\title{
Some Examples for the Automatic Generation of Self-Assessment Queries in Manufacturing Processes Engineering Subjects
}

\author{
Alfredo Sanz ${ }^{1, a}$, Ignacio González $z^{2, b}$, Félix Calvo ${ }^{3, c}$ and Eva María Andrés ${ }^{4, d}$ \\ ${ }^{1,2,3}$ Department of Aerospace Materials and Production, ETSI Aeronáutica y del Espacio, \\ Universidad Politécnica de Madrid, Plaza del Cardenal Cisneros, 3, 28040, Madrid, España \\ ${ }^{4}$ Tecnogetafe, Eric Kandel, 2, 28906, Getafe, Madrid, España \\ aa.slobera@upm.es, ${ }^{b}$ ignaciof.gonzalez@upm.es, ${ }^{c}$ felix.calvo@upm.es, \\ devamaria.andres@imdea.org
}

Keywords: Automatic generation, b-learning, questionnaires, self-evaluation, manufacturing.

\begin{abstract}
This paper presents different examples of automatic generation of queries to be implemented in a b-learning platform. Queries can be used as complementary contents and selfassessment by students in Manufacturing Processes subjects. They are useful when the available time for classroom teaching is reduced and also in blended applications, allowing students the consolidation of in-person received knowledge. Firstly, some considerations about the teaching of Manufacturing Processes subjects and about the types of queries to be generated automatically are explored. Secondly, a list of potential manufacturing topics for which this methodology is suitable is proposed, selecting three of them. Finally, and by means of non-specialized computer tools, an example of application is developed and the obtained results are presented in each topic
\end{abstract}

\section{Introduction}

Engineering education, and particularly, that related to manufacturing processes engineering, have kept a positive gradient in the number of enrolled students in Spain during the last years. This trend has been recurrent in most of centers and degrees imparting Manufacturing Processes [1]. Considering both the student number and the reduced availability of ECTS credits for the subject teaching, two problems of complex resolution may appear. The first one consists of maintaining a practical Laboratory activity in groups whose sizes are reduced enough to involve the student in the performed activity. The second one consists of the lack of time to solve practical application cases on the subjects theoretically explained, without detriment of the available time for the teaching of the theoretical syllabus. Both problems, especially the second one, are critical in the ETSI Aeronáutica y del Espacio (ETSIAE) of the Universidad Politécnica de Madrid, reason why diverse actions have been taken in order to reduce their negative effects [2]. One of them is the use of b-learning platforms, particularly Moodle, to provide resources where the student can work on his own on application examples. These examples are aimed to the self-assessment and the consolidation of the knowledge acquired in the classroom teaching and to the accomplishment of evaluation tests. For this purpose, it is necessary to generate high enough number of queries which comply with two conditions: to be adapted to every student needing and to be different enough in order to avoid their frequent repetition. The second condition is essential when the aim of the exercise resolution has an evaluation nature. The manual generation of a high number of queries involves a great effort. For this reason it is desirable to search techniques for its automation. In the following sections different examples of automatic generation are included, appearing additionally a set of ideas translatable to other contents beyond the manufacturing processes engineering itself.

\section{General considerations}

Prior to the presentation of each example, certain general considerations for the automatic generation of queries shall be highlighted, especially as far as numerical queries are concerned. Although, these observations are not always applicable, they are common in the query generation. In this section they are listed, using specific examples in order to show how to address their implementation. 
Supporting software tools. Although there is no restriction regarding this topic, it is always recommended to use simple user-friendly tools. In this regard, two stages in the query generation can be distinguished: the generation of results and the introduction of the mentioned results as a block of formulations to be exported to the b-learning software. In the work of Sanz et al [3] some alternatives are proposed and a complete procedure to create queries is detailed. The most common option is the use of two different tools, the first for the calculations and generation of the values that define every query and the second one for the query adaptation to its final format and exportation.

Range of numerical queries. To obtain the numerical results useful as input data for the related exercise, the use of a function generating random values is almost indispensable. It is important to assure that the generated data complies with a realistic range of values and these are properly rounded off to avoid confusion in the formulation. For instance, in case of using a spreadsheet, the RAND() and ROUND() functions must be conveniently used to get input data to the proposed formulation. Similarly, the ROUND() function must be applied again in order the numerical results corresponding to the exercise solution to be exactly reproduced by the student, being necessary to indicate this condition (number of significant digits of the answer) explicitly in the formulation. Even when the input data come from a table (and, hence, they are fixed values), the use of a random variable to select randomly any of the possible values of the table in each query is convenient.

Number of queries. It depends on the available range of values of each parameter and on the number of students to which the questionnaire is addressed. In general, the evaluation and selfassessment needing are widely covered if the query number is equivalent to the student's number. Depending on the considered variable, the number of available values may be lower than the number of queries. This necessarily implies that in more than a query the same value of the related variable appears. This situation only causes problems when there is a unique input variable for the query, since in case of multiple variables, the number of possible options is the cardinal of their Cartesian product. If this occurs (number of variables lower than the query number) and the selection of the values is randomly accomplished, it is convenient to filter the final results to remove duplicate queries.

Type of queries. The different b-learning platforms offer different formats to formulate queries [4]. Among all of them, and without detriment of the rest, the most adequate are the unique answer (True/False), the numerical answer and the multiple choice formats, being the latter considered the preferred one when a self-assessment activity is developed. For the evaluation it is recommended to use the second format since the student does not receive an initial hint of the possible solutions and he must obtain the correct answer without the aid of delimiting them in a finite set of values.

Generation of alternative answers. In the case of numerical multi-choice queries, both the solution and the plausible alternative answers must be defined. Regarding the numerical values of the alternative answers, a pattern which allows to recognize the correct answer without solving the proposed problem must be avoided. As an example, let us suppose that the correct solution of a query is a numerical value $\mathrm{X}$, and the proposed alternative answers are the correct solution plus a random value $r_{1},\left\{X+r_{1}\right\}$, and the correct solution minus a random value $r_{2},\left\{X-r_{2}\right\}$. In this situation, the student shall end up detecting that the correct answer is always the middle numerical value, not being obliged to solve the proposed exercise. If the answer "none of the other three" statement is also used as an alternative answer, the solution is no longer evident due to the possibility of the central value to be incorrect. However, the probability to guess the correct answer is always higher when the middle value is selected. The proposed procedure to solve this situation consists of defining a number of alternative answers higher than the number of choices which shall be presented as possible solutions to the query, and randomly choose the answers which shall accompany each formulation. Every option can be generated implementing a "wrong but plausible" solution of the exercise. Therefore, the true answer will not be identifiable through the position it takes or its relative value regarding the rest of answers. 
Rejection criteria. The use of random elements in the definition of the input variables and in the selection of answers in the case of multiple choice queries may lead to duplicities or results which, being individually valid, do not adjust properly to the set of answers. For this reason, it is convenient to apply a set of rejection criteria to each query, assuring that its use complies with the initial formulation which has generated it.

\section{Application examples}

In the selection of the type of query, the nature of its content is essential. Thus, the type of query used for mainly theoretical contents may be substantially different from the more practical ones, such as numerical applications. The Table 1 collects some groups of contents belonging to the manufacturing disciplines imparted in the ETSIAE. Groups with mainly theoretical contents, named as 'theoretical groups' have been separated from groups with mainly both, theoretical and practical (numerical applied) contents, named as 'theoretical-practical contents groups'.

Table 1. Groups of contents in the ETSIAE subjects related to Manufacturing processes

\begin{tabular}{|c|l|l|}
\hline Group & \multicolumn{1}{|c|}{ Designation } & \multicolumn{1}{|c|}{ Group Condition } \\
\hline 1 & Design and related specifications & Theoretical-practical \\
\hline 2 & Metrology & Theoretical-practical \\
\hline 3 & Fusion, casting shaping & Theoretical \\
\hline 4 & Welding and part joint shaping & Theoretical \\
\hline 5 & Sintering and additive manufacturing shaping & Theoretical \\
\hline 6 & Plastic deformation forming & Theoretical-practical \\
\hline 7 & Material removal shaping (machining) & Theoretical-practical \\
\hline 8 & Special processes and non-conventional material forming & Theoretical \\
\hline 9 & Production automation & Theoretical-practical \\
\hline
\end{tabular}

\section{Theoretical groups of contents}

This designation includes the groups $3,4,5$ and 8 in Table 1. It does not exclude the possibility of generating numerical exercises, although it is considered that theoretical descriptive concepts of the process, their advantages and disadvantages and scope prevail on them.

The procedure proposed for these topics is based on the generation of multiple choice queries from the joint of several True/False queries. In order to generate this type of queries, it is recommended to compare different types of processes and their distinctive characteristics. Using the example of the welding processes and their scope regarding the related materials, a first table of connections such as the one included in the Table 2 could be established. This table does not intend to be exhaustive, but it must include those processes and interesting materials according to the imparted subject.

Table 2. Adequacy of Welding processes and materials

\begin{tabular}{|c|c|c|c|c|c|c|}
\hline $\begin{array}{c}\text { Welding Material/ } \\
\text { Process }\end{array}$ & $\begin{array}{c}\text { Aluminium } \\
\text { and alloys }\end{array}$ & $\begin{array}{c}\text { Titanium } \\
\text { and alloys }\end{array}$ & Steels & $\begin{array}{c}\text { Magnesium } \\
\text { and alloys }\end{array}$ & $\begin{array}{c}\text { Nickel and } \\
\text { alloys }\end{array}$ & Brass \\
\hline Combustible gas welding & $\mathbf{0}$ & $\mathbf{0}$ & $\mathbf{1}$ & $\mathbf{0}$ & $\mathbf{0}$ & $\mathbf{1}$ \\
\hline Brazing & $\mathbf{0}$ & $\mathbf{0}$ & $\mathbf{1}$ & $\mathbf{0}$ & $\mathbf{0}$ & $\mathbf{1}$ \\
\hline Coated electrode arc welding & $\mathbf{0}$ & $\mathbf{0}$ & $\mathbf{1}$ & $\mathbf{0}$ & $\mathbf{0}$ & $\mathbf{0}$ \\
\hline Gas tungsten arc welding (GTAW) & $\mathbf{1}$ & $\mathbf{1}$ & $\mathbf{1}$ & $\mathbf{1}$ & $\mathbf{1}$ & $\mathbf{0}$ \\
\hline Gas metal arc welding (GMAW) & $\mathbf{1}$ & $\mathbf{1}$ & $\mathbf{1}$ & $\mathbf{1}$ & $\mathbf{1}$ & $\mathbf{0}$ \\
\hline Laser beam welding & $\mathbf{1}$ & $\mathbf{1}$ & $\mathbf{1}$ & $\mathbf{1}$ & $\mathbf{1}$ & $\mathbf{0}$ \\
\hline Resistance welding & $\mathbf{0}$ & $\mathbf{1}$ & $\mathbf{1}$ & $\mathbf{0}$ & $\mathbf{0}$ & $\mathbf{0}$ \\
\hline
\end{tabular}

In the example considered in the Table 2 , seven welding processes and six types of materials which can be or not adequate in each process are included. This implies a total of $7 * 6=42$ true combinations only by indicating which process and material is adequate according to the table, and other 42 false combinations corresponding to the opposite of the previous combinations. This means 
that, using the example of the cell $(2,2)$ shaded in the Table 2, which corresponds to 'brazing' and to 'titanium and alloys', there is a true formulation which establishes that 'The brazing is not adequate to weld titanium and its alloys', and a false formulation which establishes that 'The brazing is adequate to weld titanium and its alloys'. Thus, 84 True/False queries can be generated. These shall increase the data base of queries. Using an analogous procedure with other properties linked to the related processes, such as thickness levels or operational conditions allows to generate a high number of True/False queries which can be progressively incorporated to the queries data base.

Once a significant number of True/False questions have been created, it is possible to transform them in multi-choice queries by combining a number of True/False questions in the same formulation. Since this task may be tedious if it is performed manually, this automation is proposed:

i) A number of True/False questions equal to the number of options in the multi-choice query is randomly chosen.

ii) It must be checked that only one of the selected True/False questions has an answer contrary to the rest.

iii) If the previous check is complied, this query is valid and, on the contrary, the process is repeated from start.

iv) This process is repeated again from the step i) as many times as necessary.

In the Table 3 , the implementation of the steps i) to iv) is reproduced in case of using a spread sheet in the generation of the queries. In the code of the Table 3, the True/False questions are assumed to be in a different sheet called 'Base_Sheet' so that the question formulations are in the ' $\mathrm{X}$ ' column and the right answer, containing the letter ' $\mathrm{T}$ ' or ' $\mathrm{F}$ ', are in the ' $\mathrm{Y}$ ' column of the 'Base_Sheet'. In this situation, through the four first columns (designated in the table as rows ' $q 1$ ', ' $q 2$ ', ' $q 3$ ' and ' $q 4$ ') four random numbers between the number of available questions are chosen. These four numbers point to four True/False questions (in fact randomly chosen) from the 'Base sheet'. The limits for the random selection are included between 2 (note that the first row of the 'Base' sheet corresponds to the heading and therefore, it must not be considered) and the total number of queries determined through the COUNTA() command. The next eight columns, 'forml' to 'tf4', recover the formulations and the right answers of the selected questions from the 'Base_Sheet'. The following 'group' column generates a numerical value of two digits which shows the number of false formulations and the number of true formulations chosen in the random selection. Finally, the last 'filter' column distinguishes between the queries which are valid (one unique true or false answer) and the not valid ones (step iii) of the proposed automation).

Table 3. Code to create multiple choice queries from True/False queries

\begin{tabular}{|c|c|}
\hline Heading & Expression \\
\hline $\mathbf{q 1}$ & $=$ RANDBETWEEN $(2 ;$ COUNTA(Base_Sheet $1: 1))$ \\
\hline$q 2$ & $=$ RANDBETWEEN $(2:$ COUNTA $($ Base_Sheet! $1: 1))$ \\
\hline$q 3$ & $=$ RANDBETWEEN $(2:$ COUNTA(Base_Sheet!1:I)) \\
\hline $\mathrm{q4}$ & $=$ RANDBETWEEN $(2:$ COUNTA(Base_Sheet!1:I)) \\
\hline form1 & $=$ =INDIRECT("Base_Sheet!X"\&B1) \\
\hline form2 & =INDIRECT("Base_Sheet!X"\&B2) \\
\hline form3 & =INDIRECT("Base_Sheet!X"\&B3) \\
\hline form4 & =INDIRECT("Base_Sheet!X"\&B4) \\
\hline tf1 & $=$ =INDIRECT("Base_Sheet!Y"\&B1) \\
\hline tf 2 & $=$ =INDIRECT("Base_Sheet!Y"\&B2) \\
\hline tf3 & =INDIRECT("Base_Sheet!Y"\&B3) \\
\hline tf4 & =INDIRECT("Base_Sheet!Y" \&B4) \\
\hline group & $\begin{array}{l}=(\text { forml }=" F ")+(\text { form } 2=" F ")+(\text { form } 3=" F ")+(\text { form4="F" }) \\
+10 *((\text { forml="T" })+(\text { form2="T" })+(\text { form3=""T") })(\text { form4="T") })\end{array}$ \\
\hline filter & =IF(Type=13:"tnie":IF(Type=31;"false":"delete")) \\
\hline
\end{tabular}


In the Fig. 1 the final result of a query generated through the described procedure is shown.

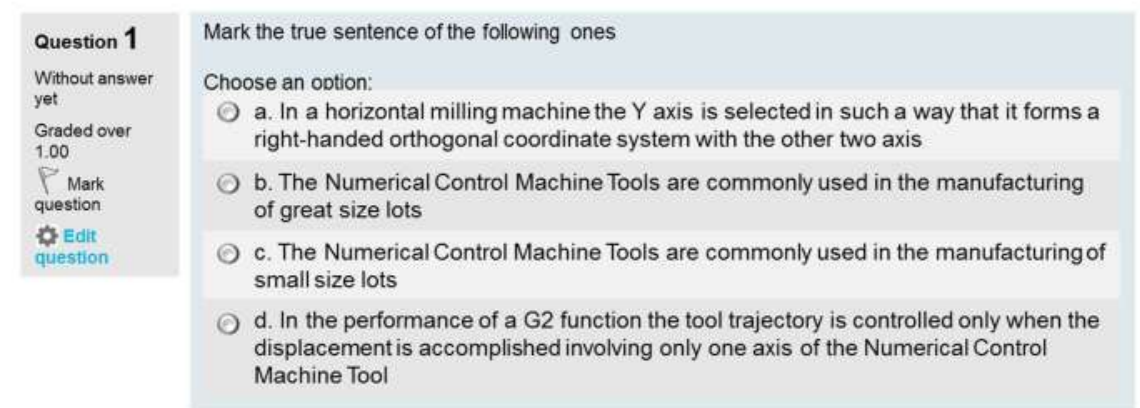

Fig. 1. Example of multiple choice query for mainly theoretical contents

\section{Theoretical-practical groups of contents}

This designation includes the groups 1, 2, 6, 7 and 9 in Table 1 and refers to the contents with immediate possibility to generate numerical exercises, which does not exclude the possibility of theoretical query generation following a similar pattern to the one described in the previous section.

Example on conventional machining. The material removal forming includes a group of processes of wide industrial diffusion. Almost all of them are liable to generate immediate numerical queries. Among the multiple existing possibilities, one related to the cutting section of a turning operation has been chosen, as it can be observed in the Fig. 2, in order to illustrate a procedure which can be easily extrapolated to other examples regarding any group of processes which require numerical resolution. In the Fig. 2 the feed $(f)$, the cutting depth $\left(a_{p}\right)$, the cutting width $(b)$, the non-deformed cutting thickness $\left(a_{c}\right)$, the position angle $\left(k_{r}\right)$ and, finally the cutting section $\left(S_{c}\right)$ are represented. The relationships among them are obtained through the expressions

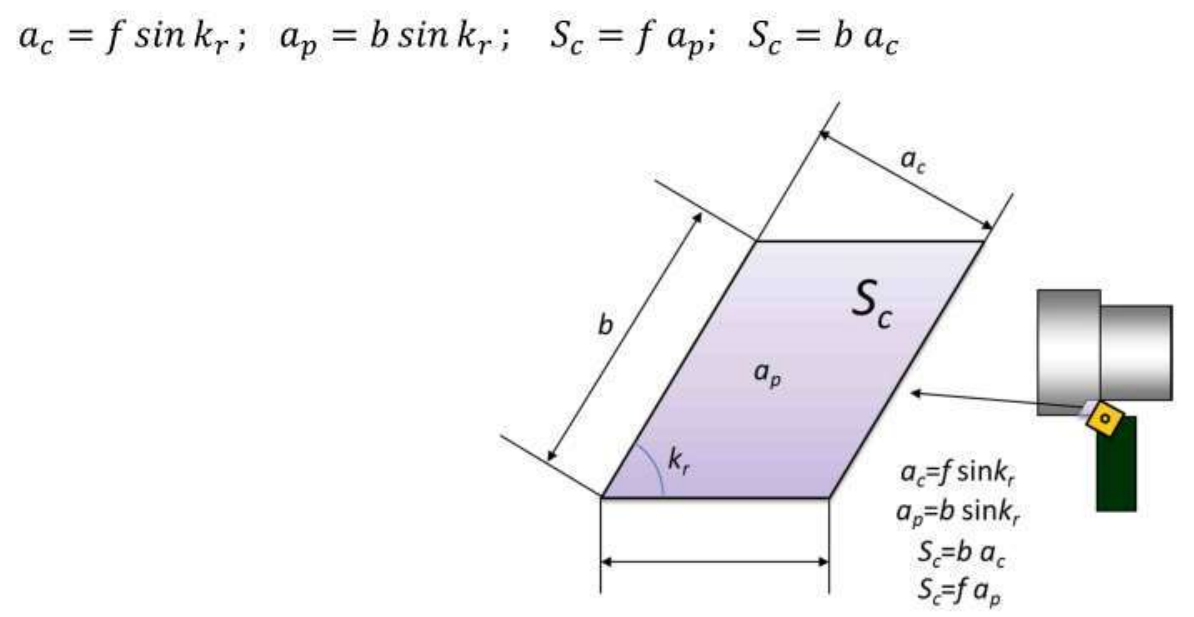

Fig. 2. Cutting section in an outer turning operation

Firstly, as described in 'Range of numerical queries', the values of each parameter must be established taking into account the round-off. In this example, there are six parameters, which are non independent, which means that, in some cases, it is only necessary to fix three of them to obtain the rest. Nevertheless, in other cases the selection of the three provided parameters can lead to a problem with no solution. If the input parameters are, for instance, $\left\{k_{r}, S_{c}, f\right\}$ it is possible to determine the other three parameters $\left\{b, a_{c}, a_{p}\right\}$. However, if the input data are $\left\{k_{r}, f, a_{c}\right\}$ in this case it is not possible to determine $\left\{b, S_{c}, a_{p}\right\}$ and the problem has no solution. Therefore, it is convenient to establish whether the cases have or not possible solution before the generation of the queries. In addition, among the former ones, it is recommended to distinguish between those cases whose solution can be reached by direct application of one of the expressions included in (1) or requires two formulae application.

For this purpose, let us consider the three-parameter set $\{$ data $\},\{$ level1 $\},\{$ level 2$\}$. The set \{data\} contains the process parameters which shall appear in the question formulation, \{level1\} 
includes the process parameters which can be directly computed from the data by exclusively applying one equation, and finally, \{level2\} stands for a process parameter which requires both, the application of a second formula and a level 1 parameter to be computed. There are twenty possible formulations when combining different parameters in the sets of \{data\}. Twelve of them correspond to formulations with solution in the level 2, four correspond to formulations with solution in the level 1 and finally, four formulations have no solution, as it is summarized in Table 4.

Table 4. Possible approaches of the query depending on the input set of the query on the material removal forming

\begin{tabular}{|c|c|c|}
\hline Solution in level 2 & Solution in level 1 & Without solution \\
\hline$\left\{k_{r}, S_{c} f f,\left\{x, a_{c}, a_{p}\right\},\{b\} /\left\{k_{r}, S_{c}, a_{p}\right\},\{b, f\},\left\{a_{c}\right\}\right.$ & $\left\{k_{r} f, a_{p}\right\},\left\{a_{c}, b, S_{c}\right\},\{\}$ & $\left\{k_{r} f, a_{c}\right\},\{\},\{\}$ \\
$\left\{k_{r} S_{c}, a_{c}\right\},\{x, f, b\},\left\{a_{p}\right\} /\left\{k_{r}, S_{c}, b\right\},\left\{a_{p}, a_{c}\right\},\{f\}$ & $\left\{k_{r}, a_{c}, b\right\},\left\{f, a_{p}, S_{c}\right\},\{\}$ & $\left\{k_{r}, a_{p}, b\right\},\{\},\{\}$ \\
$\left\{k_{r} f, b\right\},\left\{a_{c}, a_{p}, x\right\},\left\{S_{c}\right\} /\left\{k_{r}, a_{p}, a_{c}\right\},\{b, f\},\left\{S_{c}\right\}$ & $\left\{S_{c}, a_{c}\right\},\left\{a_{p}, b, k_{r}\right\},\{\}$ & $\left\{S_{c} f, a_{p}\right\},\{\},\{\}$ \\
$\left\{S_{c} f, b\right\},\left\{a_{p}, a_{c}, x\right\},\left\{k_{r}\right\} /\left\{S_{c}, a_{p}, a_{c}\right\},\{f, b\},\left\{k_{r}\right\}$ & $\left\{S_{c}, a_{p}, b\right\},\left\{f, a_{c}, k_{r}\right\},\{\}$ & $\left\{S_{c}, a_{c}, b\right\},\{\},\{\}$ \\
$\left\{f, a_{p}, a_{c}\right\},\left\{S_{c}, k_{r}, x\right\},\{b\} /\left\{f, a_{p}, b\right\},\left\{S_{c}, k_{r}\right\},\left\{a_{c}\right\}$ & & \\
$\left\{f, a_{c}, b\right\},\left\{k_{r}, x, S_{c}\right\},\left\{a_{p}\right\} /\left\{a_{p}, a_{c}, b\right\},\left\{k_{r}, S_{c}\right\},\{f\}$ & & \\
\hline
\end{tabular}

For the automatic generation of the queries, it is enough to select any set of three parameters of the level 1 or the level 2, and to establish a range of values for the input parameters. Then, for instance, if the chosen set of three parameters is $\left\{k_{r}, f, a_{p}\right\}$ and the variation range established are those in the Table 5, more than 1 million (exactly 1008518) possible queries are obtained in total with different numerical values $(1008518=41 * 98 * 251)$. Being this number significantly high, if a spreadsheet is used, it is sufficient to perform a selection of the desired number of queries using the command =RANDOMBETWEEN(Minimum;Maximum)*Round-off, taking the corresponding values from the Table 5 for each parameter.

Table 5. Value range and round-off for input parameters in the query on material removal shaping

\begin{tabular}{|c|c|c|c|c|}
\hline Parameter $\{$ symbol $\}[$ units] & Minimum & Maximum & Round-off (10 & Options \\
\hline Position angle $\left\{k_{r}\right\}\left[{ }^{\circ}\right]$ & 25 & 65 & $10^{0}$ & 41 \\
\hline Feed $\{f[\mathrm{~mm} / \mathrm{rev}]$ & 0.03 & 1.00 & $10^{-2}$ & 98 \\
\hline Cutting depth $\left\{a_{p}\right\}[\mathrm{mm}]$ & 0.05 & 3.00 & $10^{-2}$ & 251 \\
\hline
\end{tabular}

The Fig. 3 depicts the final result of a query generated through the described procedure. It must be highlighted that the proposed example leads to a numerical answer query which can be converted into a multi-answer query by adding the appropriate alternative answers. Note that it is not necessary the three-parameter set to appear in the statement. Moreover, any of the three-parameter sets of the approach included in the Table 4 can be used, which will result in a potential number above sixteen millions of different queries if it is assumed that the values established in the Table 5 are the ones used.

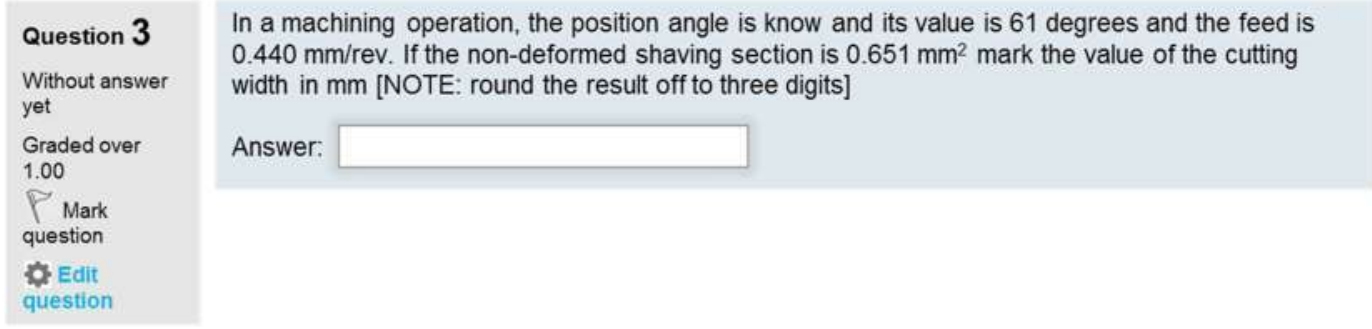

Fig. 3. Example of numerical answer query for the example of query on material removal forming

Example on design specifications and Metrology. Within this group, a great range of contents related to dimensional tolerances, operations with dimensions, geometrical tolerances, surface finish, dimensional metrology or measurement uncertainty calculation, among others, are included. The chosen example relates the interval width of the dimensional tolerance of a specification $(T)$ and the measurement uncertainty $(U)$ of the equipment used in its verification, considering the variability of the selected manufacturing process. For this purpose, the population of manufactured parts is assumed to be characterized by a known statistical distribution which allows to determine the expected proportions of right $(\alpha)$, defective $(\beta)$ or suspect $(\delta)$ parts in the established conditions. 
Fig. 4 depicts the already mentioned elements, being possible to obtain the different proportions of manufactured parts through the area enclosed by the density function $f(x)$ in the corresponding interval. If the population is assumed to be characterized by a Normal distribution $(\mu, \sigma)$ in such a way that the mean of the population coincides with the center of the tolerance interval, the part proportions can be determined through

$$
\begin{aligned}
& \alpha=\frac{1}{\sigma \sqrt{2 \pi}} \int_{x_{\text {aam }}-T / 2+U}^{x_{\text {aan }}+T / 2-U} e^{-\frac{1}{2}\left(\frac{\mu-t}{\sigma}\right)^{2}} d t \\
& \beta=2 * \frac{1}{\sigma \sqrt{2 \pi}} \int_{-\infty}^{x_{\text {nom }}-T / 2-U} e^{-\frac{1}{2}\left(\frac{\mu-t}{\sigma}\right)^{2}} d t \\
& \delta=2 * \frac{1}{\sigma \sqrt{2 \pi}} \int_{x_{\text {nom }}+T / 2-U}^{x_{\text {nom }}+T / 2+U} e^{-\frac{1}{2}\left(\frac{\mu-t}{\sigma}\right)^{2}} d t
\end{aligned}
$$

Making the population mean coincide with the nominal value of the specification and letting $F(x)$ be the function of the Normal distribution $N(0 ; 1)$ expressed through

$$
F(x)=\frac{1}{\sqrt{2 \pi}} \int_{-\infty}^{x} e^{\frac{t^{2}}{2}} d t
$$

if the stochastic variable of the expressions (2), (3) and (4) is standardized, these can be rewritten as

$$
\alpha=2 F\left(\frac{T / 2-U}{\sigma}\right)-1 ; \beta=2-2 F\left(\frac{T / 2+U}{\sigma}\right) ; \delta=1-\alpha-\beta
$$

which are the expressions to be used to solve the numerical exercises.

Following an approach analogue to the one proposed in the previous section, any numerical query in which three of the six parameters $T, U, \sigma, \alpha, \beta$ y $\delta$ are provided, has solution, except from the three-parameter set $\{\alpha, \beta, \delta\}$. The Table 6 provides some illustrative ranges for the proposed example, taking $\{T, U, \sigma\}$ as input parameters. In the mentioned ranges, conditions have been established in such a way that the tolerance/uncertainty ratio $(T / 2 U)$ and the process capacity $\left(C_{p}=T / 6 \sigma\right)$ vary in realistic margins. It can be proved that these ranges allow to achieve 17010 groups of data $\{T, U, \sigma, \alpha, \beta, \delta\}$ which, subsequently, allow the generation of 323190 queries by simply considering the combinations of the six parameters taken in sets of three elements and deducting the already mentioned parameter set $\{\alpha, \beta, \delta\}$.

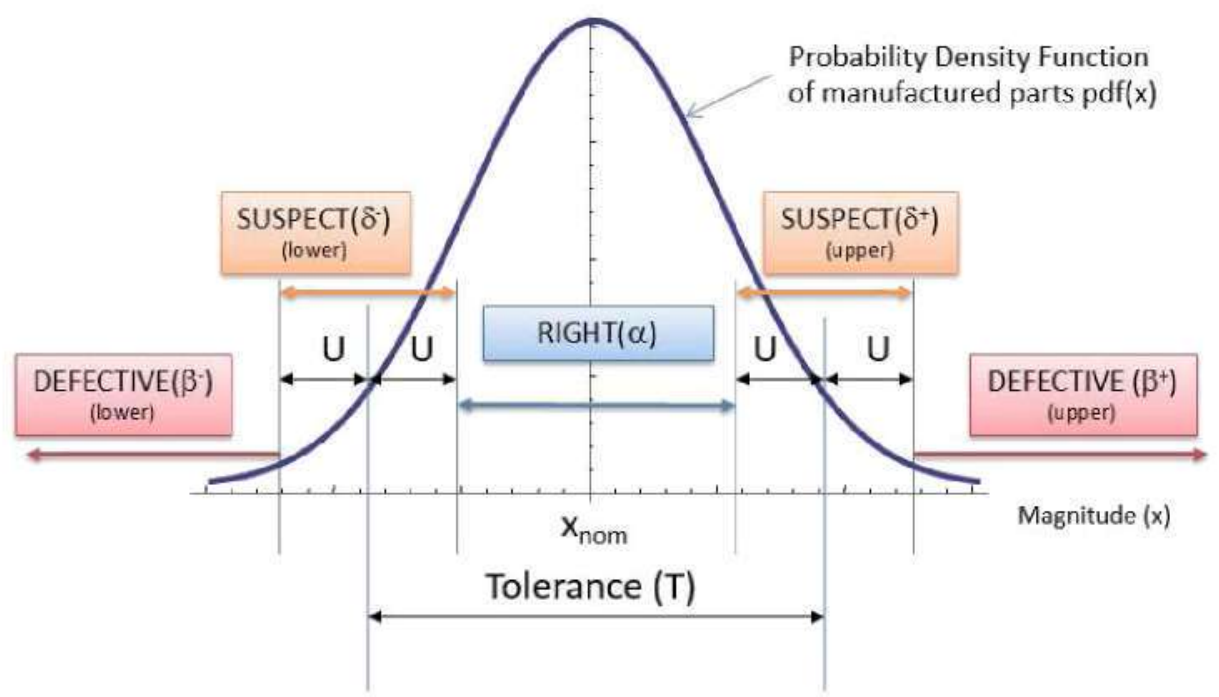

Fig. 4. Distribution of the population of manufactured parts and intervals associated to right, suspect and defective parts 
Table 6. Integer values range and units for the input parameters in the query about design specifications and metrology

\begin{tabular}{|c|c|c|c|c|}
\hline Parameter $\{$ symbol $\}$ [units] & Minimum & Maximum & Condition & Options \\
\hline Tolerance range $\{T\}[\mu \mathrm{m}]$ & 20 & 100 & & 81 \\
\hline Measurement uncertainty $\{U\}[\mu \mathrm{m}]$ & 1 & 15 & $3 \leq T / 2 U \leq 10$ & 15 \\
\hline Standard deviation $\{\sigma\}[\mu \mathrm{m}]$ & 2 & 15 & $1<T / 6 \sigma<2$ & 14 \\
\hline
\end{tabular}

In the Fig. 5 a possible formulation for the related example is included. It must be noted that it is always possible to play by using or not the tables of normal distribution or including different variations such as establishing that the tolerance $T$ derives from a standardized ISO value, requiring that the student handles the dimensional tolerance tables [5] for the resolution of the exercise.

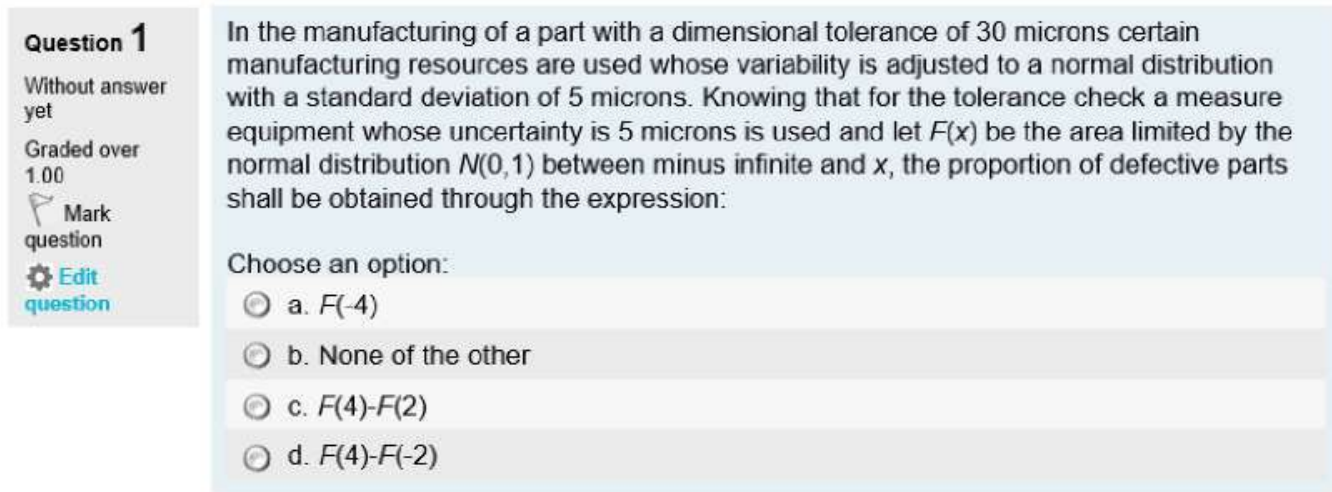

Fig. 5. Multiple choice query example about design specifications and metrology

\section{Conclusions}

When a high number of students and a reduced ECTS credit number coincide in the teaching of subjects related to the Manufacturing Processes Engineering, the availability of a high enough number of queries which allows the students the accomplishment of self-assessment exercises is very convenient, in order to consolidate the knowledge received during the theoretical classroom teaching. In the present work a set of ideas focused on the automatic generation of self-assessment queries has been proposed, particularizing them on some examples linked to subjects of the Manufacturing Processes Engineering. This is aimed to provide some guidelines for the automatic generation of queries both conceptual and numerical, based on the acquired experience which enables the generation of new formulations and helps to improve the teaching activity.

\section{References}

[1] C.S. Nair, A. Patil, P. Mertoya, Re-engineering graduate skills- a case study, European Journal of Engineering Education, 34:2, 131-139. (2009)

[2] Diez años de Proyectos de Innovación Educativa en la UPM. http://innovacioneducativa.upm.es/documentos/proyectos/informe_10_a\%C3\%B1os_ie_marzo2016 .pdf, Last access March 2017

[3] A.Sanz Lobera, I. González Requena, Generación Automática de Ejercicios de Aplicación en Asignaturas Tecnológicas, XVI Congreso Universitario de Innovación Educativa de las Enseñanzas Técnicas (CUIEET), 23-26 septiembre 2008, Cádiz. (2008)

[4] M.G. Violante, E. Vezzetti, Implementing a New Approach for the Design of an E-Learning Platform in Engineering Education, Wiley Periodicals, Inc., 2012.

[5] ISO 286-1:2010 Geometrical product specifications (GPS) -- ISO code system for tolerances on linear sizes -- Part 1: Basis of tolerances, deviations and fits 